\title{
Genetic deletion of faim reveals its role in modulating c-FLIP expression during CD95-mediated apoptosis of lymphocytes and hepatocytes
}

\author{
$\mathrm{J} \mathrm{Huo}^{1,3}, \mathrm{~S} \mathrm{Xu}{ }^{1,3}, \mathrm{~K} \mathrm{Guo}^{2}, \mathrm{Q}$ Zeng $^{2}$ and K-P Lam ${ }^{*, 1}$
}

Fas-apoptosis inhibitory molecule (FAIM) is inducibly expressed in B lymphocytes and had been shown to antagonize Fas-mediated killing of B-cell lines in vitro. However, its mechanism and role in vivo are unknown. We have generated faim ${ }^{-I}$ mice and found these mutants to be viable. In contrast to fas $^{-1-}$ mice, faim $^{-1-}$ mice have normal B- and T-cell populations. However, faim ${ }^{-1-}$ B cells and thymocytes show increased sensitivity to Fas-triggered apoptosis in vitro, and faim ${ }^{-1-}$ mice suffer greater mortality and exhibit exacerbated liver damage in response to Fas (CD95) engagement in vivo. The lack of FAIM results in greater activation of caspase- 8 and -3 in Fas-stimulated thymocytes. Detailed biochemical analyses further reveal the decreased expression of $c-F L I P(L)$ and $c-F L I P(R)$ in faim ${ }^{-1-}$ thymocytes and increased association of caspase-8 with Fas in Fas-activated mutant cells. Decreased levels of $c-F L I P(L)$ and $c-F L I P(R)$ are also evident in faim ${ }^{-1-}$ liver. Thus, FAIM plays a novel role in modulating Fas-mediated apoptosis and acts through influencing the expression of c-FLIP and regulating the physical binding of caspase-8 to Fas.

Cell Death and Differentiation (2009) 16, 1062-1070; doi:10.1038/cdd.2009.26; published online 20 March 2009

CD95 or Fas (APO-1/TNFRSF6) is a member of the tumor necrosis factor (TNF) receptor superfamily and is capable of inducing apoptosis in a variety of cell-types. ${ }^{1,2}$ The physiological ligand for Fas is CD178 (FasL), which is expressed mainly by activated T cells. ${ }^{3}$ In mice, mutation in fas (Ipr mice) or fasl ( $g / d$ mice) leads to lymphoproliferation, the accumulation of abnormal lymphocytes, autoantibody production, and this ultimately results in systemic autoimmunity. ${ }^{3,4}$ Thus, Fasmediated apoptosis is critical for regulating the function and homeostasis of lymphocytes.

Fas-signaling is triggered upon the binding of FasL, which leads to the trimerization of Fas and the formation of the downstream death-inducing signaling complex (DISC) that comprises the cytosolic adapter protein FADD and procaspase- $8 .{ }^{5}$ Procaspase- 8 undergoes auto-cleavage to generate active caspase-8, which in turn leads to the activation of caspase-3 and execution of apoptosis. As apoptosis plays an important role in physiology, its activation process must be tightly regulated. Hence, other than activators, negative regulators of Fas-signaling also exist. One of these inhibitors of Fas-mediated apoptosis is the cellular FLICE-inhibitory protein (c-FLIP) which is known to antagonize Fas-signaling by interfering with the recruitment of procaspase-8 to the DISC. ${ }^{6}$

Fas-apoptosis inhibitory molecule (FAIM) was also cloned as an inducibly expressed, anti-apoptotic protein that antagonized Fas-triggered cell death of B-cell lines in vitro. ${ }^{7}$ However, its mode of action is unknown. FAIM is highly conserved in evolution and widely expressed in all tissues although it bears no homology to other known proteins. ${ }^{7}$ Although most tissues express the short isoform of the protein (FAIM-S), neuronal cells express both FAIM-S and a long isoform, FAIM-L. ${ }^{8}$ FAIM-S was shown to promote neurite outgrowth through a mechanism involving NF- $\kappa \mathrm{B}$ and Ras-ERK activation, ${ }^{8}$ whereas FAIM-L was demonstrated to protect neurons from cell death in response to Fas and TNFR1 engagement. ${ }^{9}$

Apart from its role in protecting B-lymphocytes and neuronal cells from death receptor-induced apoptosis, ${ }^{7,9}$ FAIM was also shown to improve the survival of HEK-293 cells in serum-free media ${ }^{10}$ and $\mathrm{CHO}$ cells in fed-batch cultures. ${ }^{11}$ This suggests a potential application of FAIM in biotechnology through its role in enhancing the longevity of cell lines used for the production of biologics.

In this study, we examined the role of FAIM in vivo by gene knockout in mice. We found faim $^{-1-}$ mice to be viable. However, faim $^{-1-}$ B cells, thymocytes and hepatocytes showed increased sensitivity to Fas-triggered apoptosis, and faim $^{-1-}$ mice showed exacerbated lethality and liver damage in response to Fas treatment in vivo. The lack of FAIM resulted in increased activation of caspase- 8 and -3 in thymocytes upon Fas stimulation and this was probably due to the decreased expression of c-FLIP and increased physical association of caspase- 8 with Fas in these mutant cells.

\footnotetext{
${ }^{1}$ Laboratory of Immunology, Bioprocessing Technology Institute, Agency for Science, Technology and Research (A*STAR), Singapore and ${ }^{2}$ Laboratory of Cancer Metastasis, Institute of Molecular and Cell Biology, Agency for Science, Technology and Research (A*STAR), Singapore

${ }^{*}$ Corresponding author: K-P Lam, Laboratory of Immunology, Bioprocessing Technology Institute, Agency for Science, Technology and Research, No. 06-01, Centros, 20 Biopolis Way, Singapore 138668, Singapore. Tel: + 65 64070001; Fax: + 65 64789561; E-mail: lam_kong_peng@bti.a-star.edu.sg

${ }^{3}$ These authors contributed equally to this work.

Keywords: Fas; c-FLIP; apoptosis; caspase

Abbreviations: BCR, B-cell receptor; c-FLIP, cellular FLICE-like inhibitory protein; DEX, dexamethasone; DISC, death-inducing signaling complex; FADD, Fasassociated protein with death domain; FAIM, Fas-apoptosis inhibitory molecule; GADD45 $\beta$, growth arrest and DNA damage inducible protein $\beta$; PARP, poly (ADPribose) polymerase; $\mathrm{PI}$, propidium iodide; TNF, tumor necrosis factor; XIAP, $\mathrm{X}$ chromosome-linked inhibitor of apoptosis

Received 06.8.08; revised 05.2.09; accepted 16.2.09; Edited by P Vandenabeele; published online 20.3.09
} 


\section{Results}

Normal B- and T-cell development in faim $^{-1-}$ mice. As FAIM was shown to antagonize Fas-mediated killing of B-cell lines in vitro, ${ }^{7}$ we were interested to determine its role in vivo. We therefore inactivated faim by deleting its first two exons and starting ATG codon in mouse embryonic stem cells (Figure 1a). The deletion of faim was verified by Southern blot and PCR analyses using targeted ES cells and mouse tail DNA (Figure $1 \mathrm{~b}$ and $\mathrm{c}$ ). Western blot analyses further confirmed that neither the long nor short isoform of FAIM nor a truncated protein was detected in cell lysates obtained from mutant thymus and cerebellum (Figure 1d and e).

We found that faim $^{-1-}$ mice were viable and born in a Mendelian ratio despite the ubiquitous expression of FAIM. The life span of faim $^{-1-}$ mice was also normal as these mice did not show early mortality. This indicates that FAIM is not essential for mouse development or longevity.

As FAIM was highly expressed in spleen and thymus, ${ }^{7,8}$ we characterized B- and T-cell development in $\mathrm{faim}^{-1-}$ mice. Flow cytometry analyses revealed that mutant mice had normal $B$ and $T$ lymphopoiesis (Figure 2 ). The fractions of pro$\mathrm{B} /$ pre-B $\left(\mathrm{B}^{2} 20^{+} \mathrm{IgM}^{-}\right)$, immature $\left(\mathrm{B}^{2} 20^{+} \operatorname{lgM}^{+}\right)$and mature
$\left(\mathrm{B}^{2} 20^{\text {high }} \operatorname{lgM}^{+}\right)$B cells in the bone marrow of faim $^{-1-}$ mice were indistinguishable from those found in age- and gendermatched littermate controls (Figure 2a). Likewise, developing thymocytes at different stages of maturation such as the CD4/CD8 double-positive and single CD4 ${ }^{+}$or $\mathrm{CD}^{+}$cells were equivalently represented in faim $^{-1-}$ and wild-type mice (Figure 2b). Peripheral B- and T-cell populations in the spleen and lymph nodes were also not perturbed in the absence of FAIM. The fractions of $\mathrm{CD}_{19}{ }^{+}$or $\mathrm{B}_{220}{ }^{+} \mathrm{IgM}^{+}$peripheral $\mathrm{B}$ cells (Figure $2 \mathrm{c}$ and $\mathrm{d}$ ) or follicular $\left(\operatorname{IgM}^{+} \mathrm{CD} 21^{\text {int }} \mathrm{CD} 23^{+}\right)$and marginal zone $\left(\operatorname{IgM}^{+} \mathrm{CD} 21^{\text {high }} \mathrm{CD}^{-} 3^{-}\right)$B cells (data not shown) were comparable between wild-type and mutant animals. There was also no abnormal or skewed development of $\mathrm{CD}^{+} \mathrm{B}-1$ cells in the peritoneal cavity of faim $^{-1-}$ mice (data not shown). Detailed analyses also revealed no differences in the composition or phenotype of peripheral $\mathrm{T}$ cells as $\mathrm{CD}^{+}$or $\mathrm{CD}^{+}{ }^{+}$and $\mathrm{CD}^{+}{ }^{+} \mathrm{T}$ cells or $\mathrm{CD} 4{ }^{+}$ $\mathrm{CD}_{25}{ }^{+} \mathrm{CD} 62 \mathrm{~L}^{+}$T-regulatory and $\mathrm{CD} 4{ }^{+} \mathrm{CD} 44^{\text {high }}{ }^{\mathrm{CD}} 62 \mathrm{~L}^{\text {low }}$ T-memory/effector cells were present in similar frequencies and numbers in faim $^{-1-}$ and wild-type mice (Figure 2c, $d$ and data not shown).

As there was no gross alteration in the lymphoid populations in faim $^{-1-}$ mice, we examined the levels of serum immunoglobulins in these animals by ELISA and found that
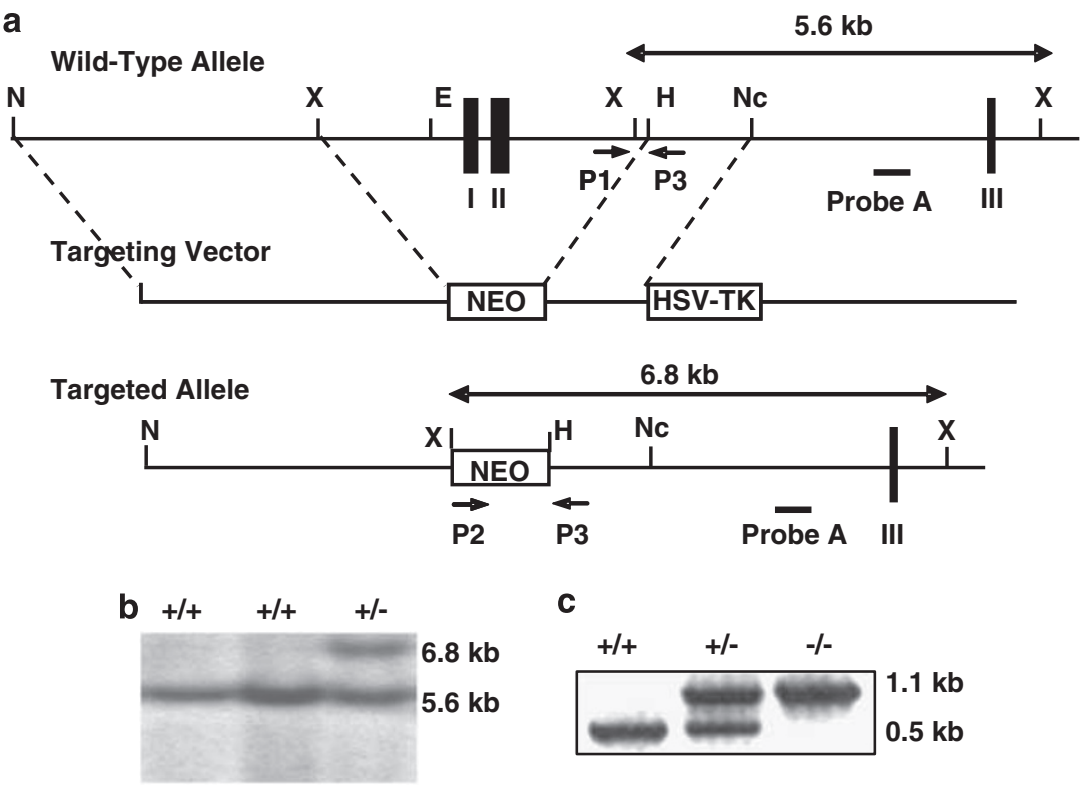

C
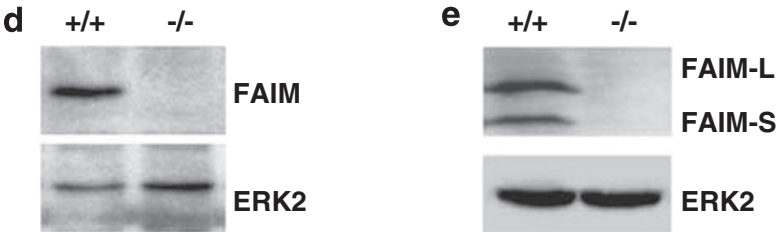

Figure 1 Generation of faim ${ }^{-1-}$ mice. (a) Partial endonuclease restriction maps of the wild-type faim locus, targeting vector and inactivated locus after homologous recombination are shown. Restriction enzyme sites are: E, EcoRI; H, Hind III; N, Notl; Nc, Ncol; and X, Xbal. The black box represents exon. Xbal digestion of genomic DNA yielded fragments of 5.6 and $6.8 \mathrm{~kb}$ for wild-type and targeted alleles, respectively, as revealed by external probe A. (b) Southern blot analysis of $X b a l-d i g e s t e d ~ D N A$ from wildtype $(+/+)$ and targeted $(+/-)$ ES cells using probe A. (c) PCR analysis of tail DNA prepared from wild-type $(+/+)$, heterozygous $(+/-)$ and homozygous $(-/-)$ FAIMknockout mice using primers P1, P2 and P3 as indicated in (a). Western blot analysis of FAIM protein expression using cell lysates from (d) thymocytes and (e) cerebellum of wild-type $(+/+)$ and homozygous $(-/-)$ FAIM-knockout mice. Anti-ERK2 blots were included as a loading control. Data shown are representative of $3-5$ independent experiments 

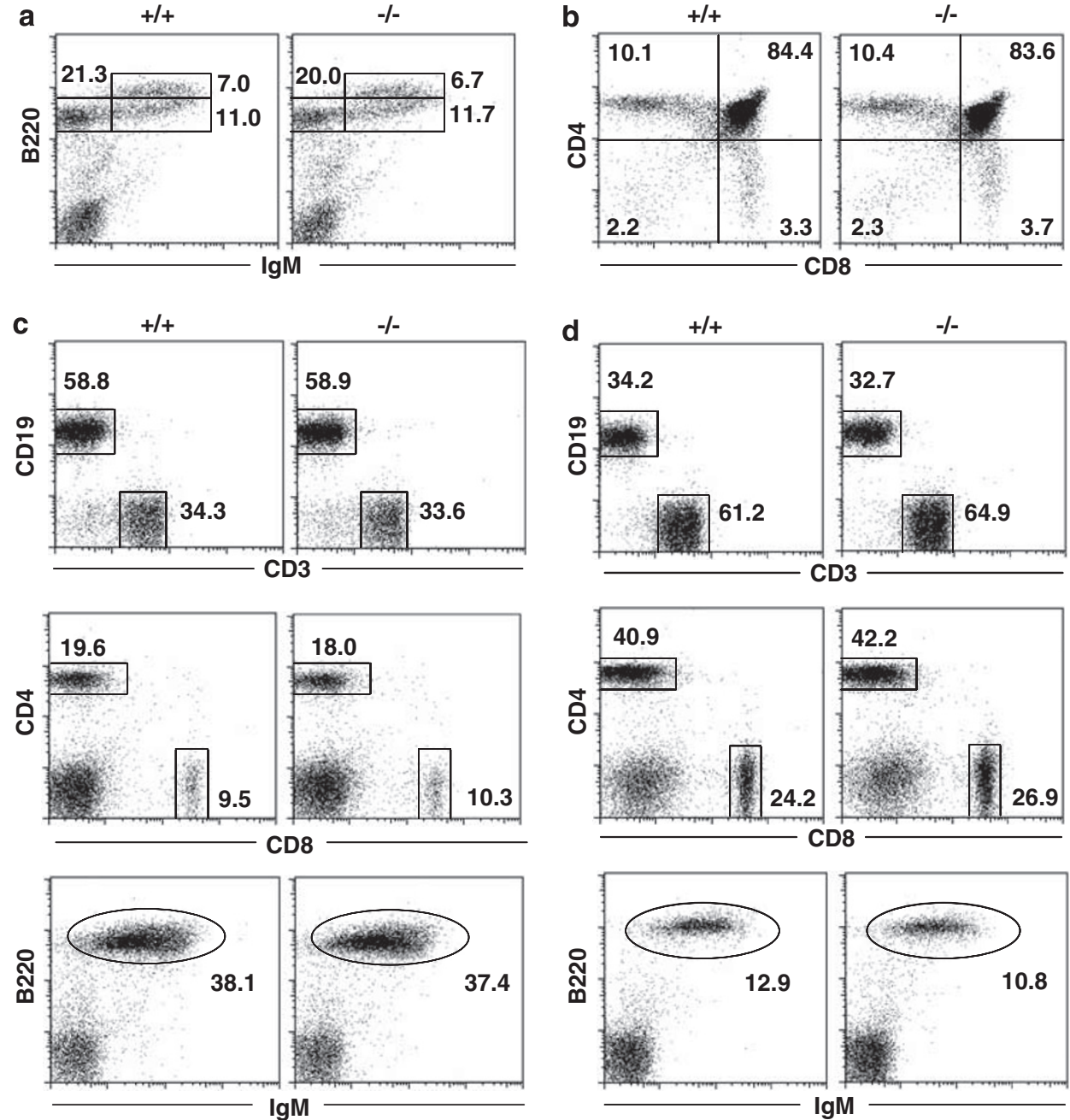

Figure 2 Flow cytometry analyses of immune cells in faim ${ }^{-1-}$ mice. FACS analyses of cells obtained from (a) bone marrow, (b) thymus, (c) spleen, and (d) inguinal lymph nodes of wild-type and faim ${ }^{-1-}$ mice using fluorochrome-conjugated antibodies. Numbers indicate percentage of lymphocytes. Data shown are representative of more than 10 independent experiments

they had equivalent amounts of $\lg M, \lg G 1, \lg G 2 a$ and $\lg G 3$ antibodies compared with wild-type animals (data not shown). Thus, the overall data indicated that FAIM was dispensable for the generation of $B$ and $T$ lymphocytes.

Faim $^{-1-}$ B cells are more susceptible to activationinduced cell death. In the absence of a drastic phenotype in $\mathrm{faim}^{-1-}$ mice, we questioned whether FAIM played a role in B-cell apoptosis as suggested by the overexpression studies in cell lines. ${ }^{7}$ To address this, we purified splenic B cells from wild-type and faim $^{-1-}$ mice and stimulated them with anti-CD40 antibodies. This treatment resulted in the expression of Fas in B cells and rendered them susceptible to FasL-triggered apoptosis unless the cells received a signal through the B-cell receptor (BCR). ${ }^{12,13}$ As shown in Figure 3a, wild-type and faim $^{-1-} \mathrm{B}$ cells, once activated by CD40 engagement, were equally susceptible to apoptosis and undergo cell death in a FasL dosage-dependent manner. The addition of anti-IgM antibodies that stimulated the BCR, to anti-CD40-treated wild-type B cells rendered these cells resistant to Fas-mediated cell death as there was no appreciable increase in the percentage of cells with DNA fragmentation in the sample treated with FasL compared with the untreated sample (37.9 versus $36.4 \%$, Figure $3 b$ ). This could partially be explained by the higher level of FAIM expression that conferred a greater level of protection from apoptosis in the anti-IgM-stimulated wild-type B-cell sample (Figure 3c). By contrast, anti-IgM stimulation did not efficiently protect anti-CD40-treated faim $^{-1-}$ B cells from FasL-triggered apoptosis as there was a significant increase in the percentage of cells with DNA fragmentation in the FasL-treated sample compared to the control (49.3 versus $35.8 \%$ ). Hence, FAIM plays a role in BCR-mediated protection of activated primary $\mathrm{B}$ cells from Fas-induced cell death.

Thymocytes lacking FAIM show enhanced susceptibility to Fas- but not dexamethasone-, $\gamma$-irradiation- or TNF- $\alpha$ induced apoptosis. It was shown previously that $\mathrm{CD} 4^{+}$ $\mathrm{CD}^{+}$thymocytes were sensitive to Fas-mediated apoptosis. ${ }^{14}$ As FAIM was also highly expressed in thymus, ${ }^{7}$ we examined if Fas-triggered apoptosis would be affected in faim $^{-1-}$ thymocytes. 

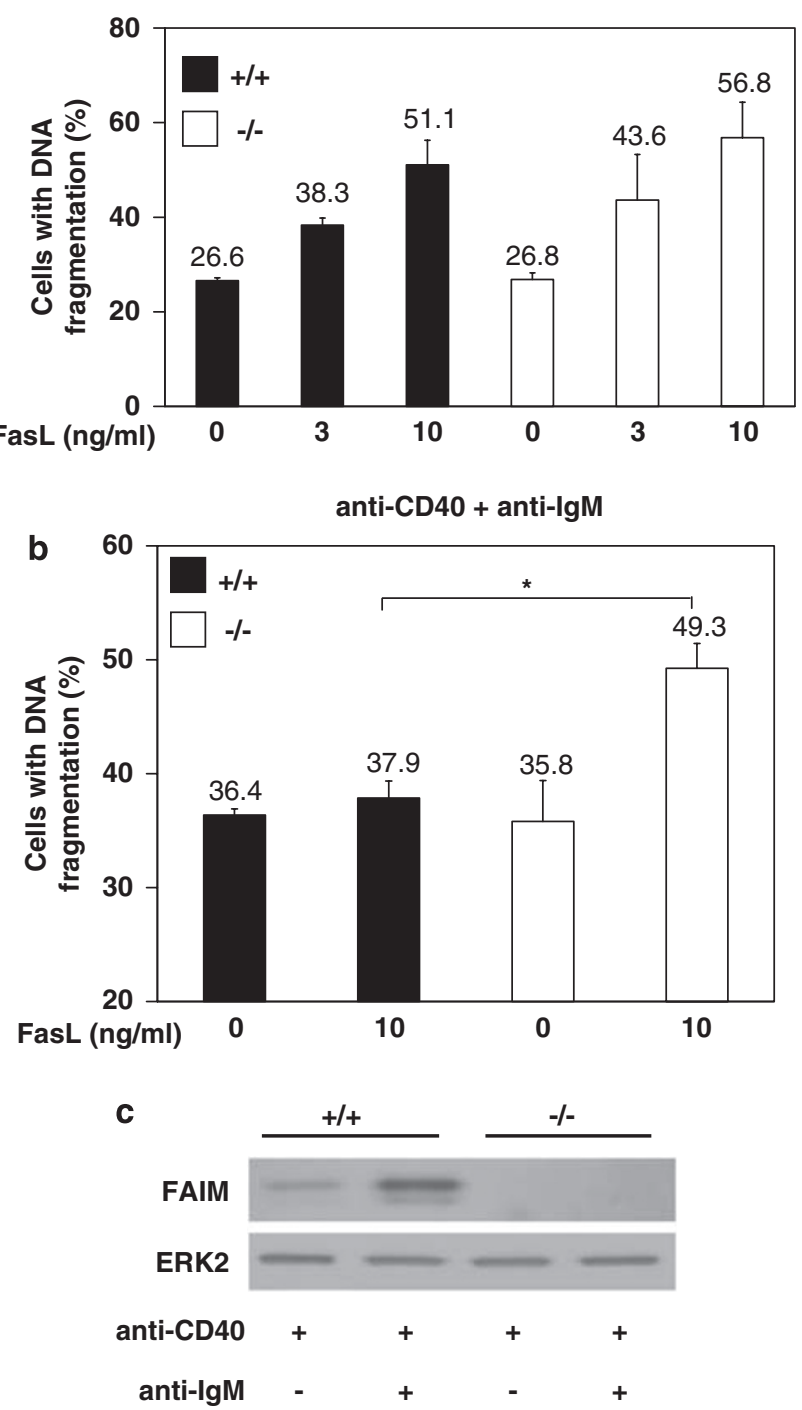

Figure 3 Anti-IgM engagement could not prevent FasL-triggered apoptosis of anti-CD40-treated faim ${ }^{-1-}$ B cells. (a) Wild-type and faim ${ }^{-1-}$ B cells were equally susceptible to FasL-triggered apoptosis after CD40 stimulation. Cells were preactivated with anti-CD40 antibodies followed by treatment with various doses of FasL as indicated. (b) Anti-IgM stimulation prevents anti-CD40-activated wild-type but not faim ${ }^{-1-}$ B cells from further FasL-triggered apoptosis. Apoptosis was analyzed by propidium iodide staining of cellular DNA content and shown as percentage of sub-G1 to the whole population. Numbers indicate mean percentage of cells with DNA fragmentation \pm S.D. Data shown are representative of five independent experiments. ${ }^{*} P=0.033$ (two-sided Student's $t$-test). (c) Western blot analysis showing FAIM protein expression in anti-CD40-activated wild-type B cells treated with anti-lgM antibody over non-treated control. The anti-ERK2 blot served as loading control. Data shown are representative of 3 independent experiments

As shown in Figure 4a, treatment of wild-type thymocytes with FasL led to increased apoptosis of these cells over nontreated control as there was an increase in the proportion of cells in sub-G1 and correspondingly, a decrease of cells in G1 phase after $6 \mathrm{~h}$ of stimulation. The same phenomenon was observed with faim $^{-1-}$ thymocytes and more significantly, the proportion of cells in sub-G1 phase in FasL-treated faim ${ }^{-1-}$ sample was much larger compared with similarly treated wild-type control. Quantitative analysis shown in Figure $4 \mathrm{~b}$ revealed that the percentage of cells with DNA fragmentation during Fasmediated apoptosis was significantly increased in faim $^{-1-}$ thymocytes compared with wild-type cells (76.1 versus $53.8 \%$ ). These data indicate that thymocytes lacking FAIM were also more susceptible to FasL-triggered apoptosis.

Thymocytes also undergo apoptosis in response to steroid, radiation and TNF- $\alpha$ treatment. ${ }^{15,16}$ To determine if faim $^{-1-}$ thymocytes would exhibit enhanced apoptosis to these stimuli, we first separately treated wild-type and mutant thymocytes with dexamethasone or subjected them to $\gamma$-irradiation. As shown in Figure 4c, faim $^{-1-}$ thymocytes did not show enhanced apoptosis to these treatments when compared with similarly treated wild-type control. Next, we treated wild-type and faim $^{-1-}$ thymocytes with recombinant TNF- $\alpha$ and again, there was no difference in the susceptibility of these cells to TNF- $\alpha$-induced apoptosis (Figure $4 d$ ). This was further substantiated at the molecular level where wildtype and faim $^{-1-}$ thymocytes showed a comparable level of $\mathrm{NF} \kappa \mathrm{B}$ activation, as evident by the similar levels of NF- $\kappa \mathrm{B}$ p65 phosphorylation and $\mathrm{l} \kappa \mathrm{B} \alpha$ degradation in the wild-type and mutant samples, upon TNF- $\alpha$ treatment (Figure 4e). Thus, FAIM acts in a specific manner to protect thymocytes from Fas-mediated but not dexamethasone- or $\gamma$-irradiation or TNF- $\alpha$ induced apoptosis.

Faim $^{-1-}$ thymocytes exhibited enhanced activation of caspase-8 and -3 upon Fas engagement. Engagement of Fas is known to activate the initiator caspase-8, and subsequently, the executor caspase-3. ${ }^{17}$ To determine if the enhanced susceptibility of faim $^{-/-}$thymocytes to Fasmediated cell death could be correlated with known molecular events in apoptosis, we directly assayed for the activation of caspase- 8 and caspase- 3 in these cells. As shown in Figure $5 \mathrm{a}$, the cleaved form of caspase- 8 was increased in wild-type thymocytes after $6 \mathrm{~h}$ of FasL stimulation. Consistent with the cellular data above, there was a significantly greater level of caspase- 8 activation in faim $^{-1-}$ thymocytes that were stimulated for the same period of time. As a consequence of the higher level of caspase-8 induction, the activation of caspase-3, as shown by the presence of its cleaved form, was also enhanced in faim $^{-1-}$ thymocytes. As a hallmark event of apoptosis, caspase-3mediated cleavage of poly (ADP-ribose) polymerase (PARP) was also increased in faim $^{-1-}$ thymocytes compared with wild-type cells after FasL treatment. The enhanced Fasmediated apoptosis and the higher induction of caspase-8 and caspase- 3 activation in faim $^{-1-}$ thymocytes were not due to altered cell surface expression of Fas as flow cytometry analysis revealed no difference in Fas expression in wildtype and faim $^{-1-}$ cells (data not shown). Thus, the data indicate that FAIM plays an inhibitory role in Fas-triggered apoptosis of thymocytes and in its absence, thymocytes exhibit enhanced cell death with increased activation of caspase- 8 and caspase- 3 in response to FasL treatment.

Impaired expression of c-FLIP(L) and c-FLIP(R) and increased physical association of caspase-8 with activated Fas in faim $^{-1-}$ thymocytes. The data above suggested that the absence of FAIM led to a defect that was downstream of Fas and resulted in an augmented activation of 


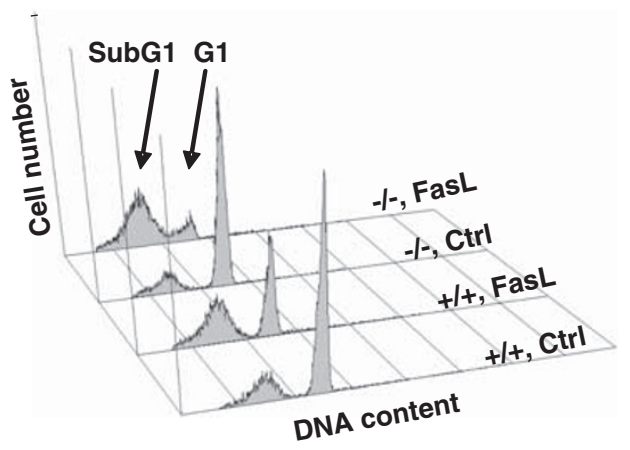

b
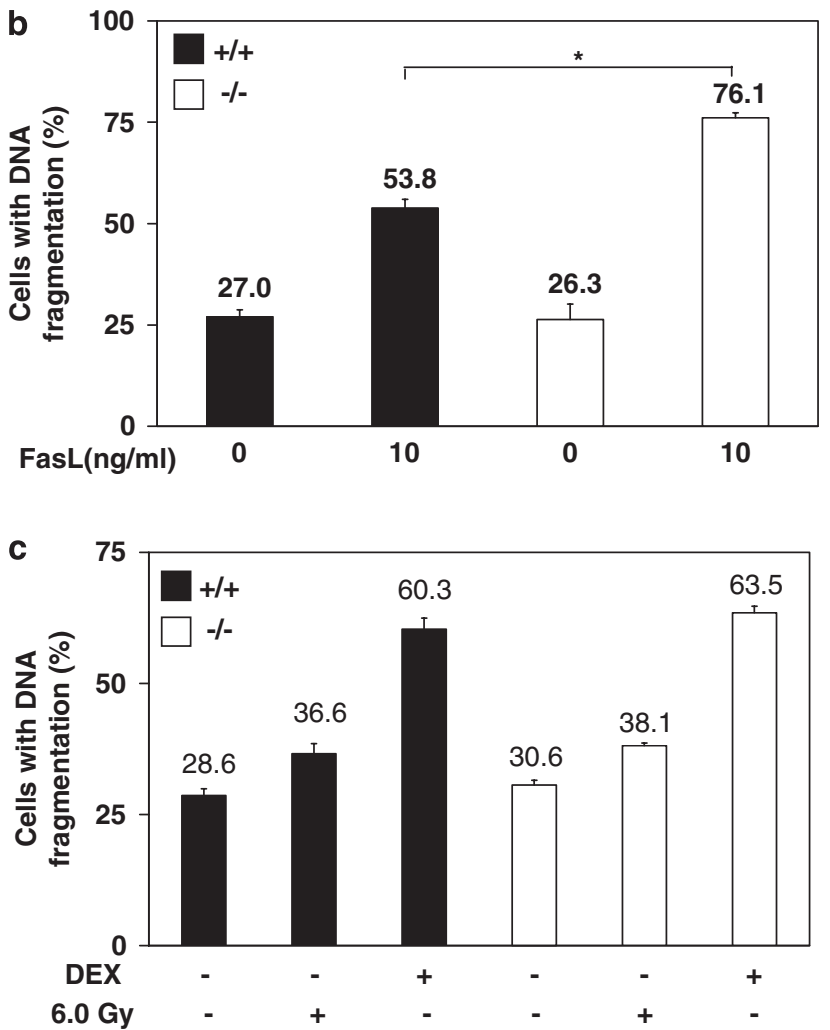

d

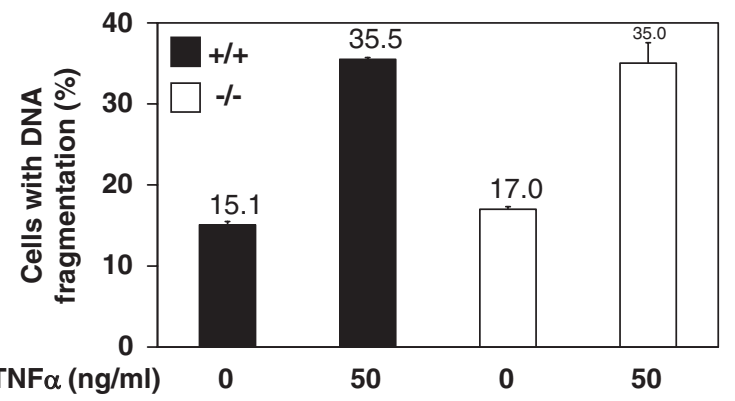

e

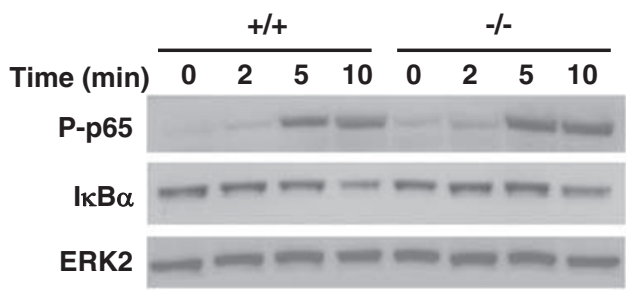

caspase-8 during Fas signaling. In an attempt to understand the mechanism whereby FAIM could inhibit Fas-mediated apoptosis, we examined the protein expression levels of $F A D D, c-F L I P(L)$ (the long isoform of c-FLIP), c-FLIP(R) (the short c-FLIP isoform expressed in mice ${ }^{18}$ ), Bcl-xL, XIAP and caspase-8 in faim $^{-1-}$ thymocytes. Some of these molecules, such as C-FLIP, FADD and caspase-8, are part of the DISC and are known to physically associate with Fas. ${ }^{5}$ Western blot analyses of whole-cell lysates indicated that there was no significant difference in the expression level of $\mathrm{Bcl}-\mathrm{xL}, \mathrm{XIAP}$, FADD and caspase- 8 between wild-type and faim $^{-1-}$ thymocytes (Figure $5 b)$. Interestingly, the amount of c-FLIP $(L)$ and c-FLIP(R) were significantly reduced in mutant thymocytes, suggesting that the increased sensitivity of faim $^{-1-}$ thymocytes to Fas-induced apoptosis could be due to a decreased expression of C-FLIP.

It was previously shown that c-FLIP could interfere with the recruitment of caspase- 8 to the DISC ${ }^{6}$ and as caspase- 8 could physically associate with Fas, ${ }^{19}$ we wondered if the reduced level of C-FLIP expression could affect the level of caspase-8 association with Fas upon its binding of FasL in faim $^{-/-}$ thymocytes. We therefore immunoprecipitated Fas or caspase8 from FasL-treated wild-type and faim $^{-1-}$ thymocytes and examined the amount of caspase-8 or Fas that was present, respectively. We chose an early time point at $10 \mathrm{~min}$ after FasL treatment ${ }^{20}$ to examine the interaction between Fas and caspase-8 in wild-type and faim $^{-1-}$ thymocytes. As shown in Figure 5c, a greater amount of caspase-8 or Fas could be co-immunoprecipitated with Fas or caspase-8, respectively in FasL-treated faim $^{-1-}$ thymocytes compared with similarly treated wild-type control. Taken together, the data suggested that in the absence of FAIM there was a reduced expression of $c-F L I P(L)$ and c-FLIP $(R)$ and this could result in greater physical association of caspase-8 with Fas during activation, which in turn could lead to the increased activation of caspases-8 and -3 and increased apoptosis in the mutant cells.

FAIM confers protection against Fas-induced liver damage. We also tested the effect of a loss of FAIM at the organism level by the in vivo administration of an agonistic anti-Fas antibody, Jo2. This treatment will lead to acute fulminant hepatitis, initiated as a result of the engagement of Fas on hepatocytes, and will result in animal death within

Figure 4 Thymocytes lacking FAIM exhibit increased apoptosis upon FasL but not dexamethasone, $\gamma$-irradiation or TNF- $\alpha$ treatment. (a) Flow cytometry analysis of FasL-induced apoptosis of wild-type and faim ${ }^{-1-}$ thymocytes. Cells were stimulated for $6 \mathrm{~h}$ with FasL (10 $\mathrm{ng} / \mathrm{ml})$, and the $\mathrm{G} 1$ and sub-G1 (apoptotic) fractions, as defined by propidium iodide staining of cellular DNA content, were indicated. Data shown are representative of six independent experiments. Graphic representations depicting percentage of cells with DNA fragmentation in wild-type and faim ${ }^{-1-}$ thymocyte samples upon FasL $(10 \mathrm{ng} / \mathrm{ml}, 6 \mathrm{~h})(\mathrm{b})$ or dexamethasone $(1 \mu \mathrm{M}, 6 \mathrm{~h})$ or $\gamma$-irradiation $(6.0 \mathrm{~Gy})$ treatment (c) or TNF- $\alpha(50 \mathrm{ng} / \mathrm{ml}$ with $100 \mu \mathrm{M} \mathrm{CHX)} \mathrm{(d)}$ treatment. Numbers indicate mean percentage of cells with DNA fragmentation \pm S.D. Data shown are representative of $3-5$ independent experiments. ${ }^{*} P=0.0001$ (two-sided Student's $t$-test). (e) Comparable TNF- $\alpha$-induced-NF- $\kappa \mathrm{B}$ activation in wild-type and faim $^{-1-}$ thymocytes. Thymocytes were treated with $10 \mathrm{ng} / \mathrm{ml} \mathrm{TNF}-\alpha$ for different time points indicated in the figure. Western blot analyses NF- $\kappa \mathrm{B}$ p 65 phosphorylation and $\mathrm{I}_{\kappa} \mathrm{B} \alpha$ degradation. Anti-ERK2 blots were used as loading controls. Data are representative of four independent experiments 
a

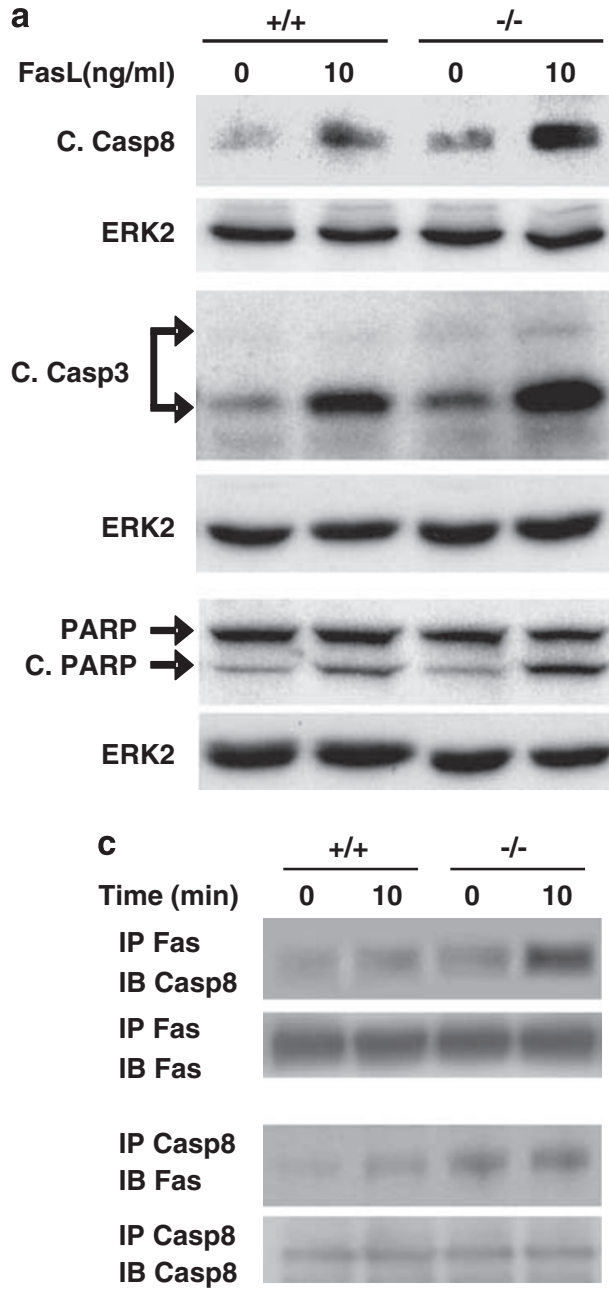

b
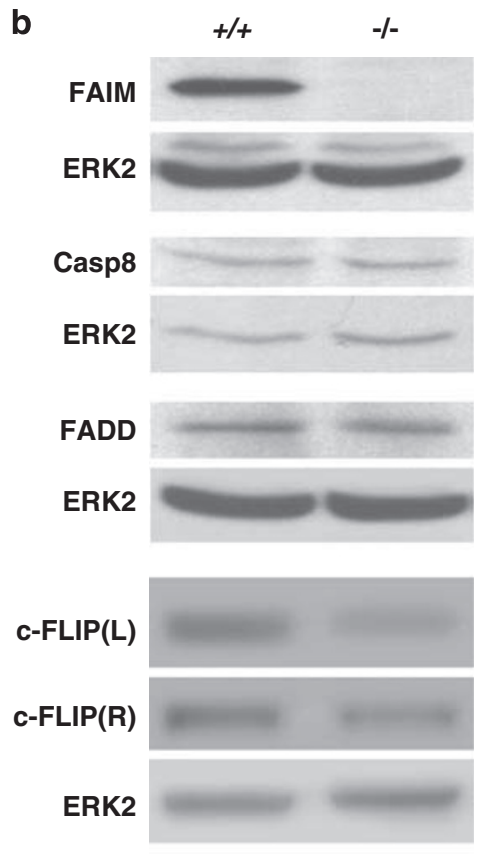

Bcl-xL

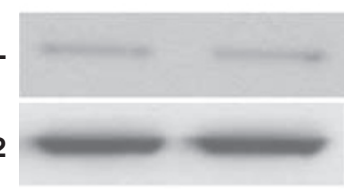

XIAP

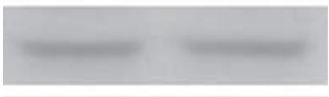

ERK2

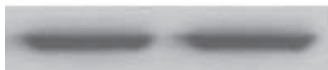

Figure 5 Biochemical analyses of caspase activation and expression of downstream Fas-signaling proteins in faim ${ }^{-1-}$ thymocytes. (a) faim ${ }^{-1-}$ thymocytes exhibit enhanced activation of caspases-8 and -3 upon FasL treatment. Western blot analyses of caspase-8 and caspase-3 activation and PARP cleavage in FasL-treated wild-type and faim ${ }^{-1-}$ thymocytes. Cells were non-treated or treated with FasL $(10 \mathrm{ng} / \mathrm{ml})$ for $6 \mathrm{~h}$ and cell lysates were probed for the cleaved forms of caspase-8, caspase-3 and PARP. (b) Reduced c-FLIP $(\mathrm{L})$ and c-FLIP $(\mathrm{R})$ expression in faim ${ }^{-1-}$ thymocytes. Western blot analyses of caspase-8, FADD and c-FLIP $(\mathrm{L}), \mathrm{c}-\mathrm{FLIP}(\mathrm{R}), \mathrm{Bcl}-\mathrm{xL}$ and XIAP expressions in wild-type $(+/+)$ and faim ${ }^{-1-}$ thymocytes. (c) Augmented Fas-caspase-8 association in FasL- $(10 \mathrm{ng} / \mathrm{ml}, 10 \mathrm{~min})$ treated faim ${ }^{-1-}$ thymocytes. Wild-type and faim ${ }^{-l-}$ thymocytes were treated with FasL and cell lysates were immunoprecipitated with anti-Fas or anti-caspase-8 and probed with anti-caspase-8 or anti-Fas antibody. The blot was subsequently reprobed with anti-Fas or anti-caspase-8 antibody to ensure pull-down of an equivalent amount of Fas or caspase-8 protein. Anti-ERK2 blots were used as loading controls. Data are representative of 3-6 independent experiments

hours. ${ }^{21}$ We injected Jo2 antibodies into wild-type and faim $^{-/-}$ mice and monitored their survival over a period of time. As shown in Figure $6 \mathrm{a}$, faim $^{-1-}$ mice succumbed to death rapidly and within $7 \mathrm{~h}$ of Jo2 injection, none of the animals survived. By contrast, although wild-type mice were also susceptible to Fasinduced death, their mortality rate was much lower and at the $7 \mathrm{~h}$ time point, at least $50 \%$ of them survived.

Next, we examined the livers of Jo2 mAb-treated mice and we chose the $2.5 \mathrm{~h}$ post-injection time point for the inspection as it was just before the occurrence of any death. As seen in Figure $6 b$, the liver sample from Jo2-injected faim $^{-1-}$ mice ( $n=13)$ had already turned dark red at this time point, which was indicative of wide-spread hemorrhage. By contrast, the liver from wild-type mice $(n=16)$ did not show any color change at this time point. Histological examination of liver sections further revealed that extensive hepatic apoptosis and hemorrhage had occurred in faim $^{-1-}$ but not wild-type mice (Figure 6c). And consistent with the observation of massive liver destruction in Jo2-injected faim $^{-1-}$ mice, there was also a significant increase in the level of alanine aminotransferase (ALT) in the sera of these mice compared to the sera of Jo2-injected wild-type or non-injected wild-type and faim $^{-1-}$ mice (Figure 6d).

Similar to our study with faim $^{-1-}$ thymocytes (Figure 5b), we also checked if the expression levels of various anti-apoptotic molecules, such as c-FLIP(L), c-FLIP(R), Bcl-xL and XIAP were perturbed in the mutant hepatocytes. As shown in Figure $6 e$, both c-FLIP $(\mathrm{L})$ and $c-F L I P(R)$ but not $B c l-x L$ or XIAP were reduced in faim $^{-1-}$ compared with wild-type liver cells, suggesting that FAIM also modulates c-FLIP expression level in hepatocytes.

Lack of increased C-FLIP expression upon BCR engagement of CD40-activated faim $^{-1}$ B cells. Given that BCR engagement could induce FAIM expression, ${ }^{7}$ and 
lead to the protection of CD40-activated wildtype but not faim $^{-1-}$ B cells from Fas-mediated apoptosis (Figure 3), and FAIM appears to regulate c-FLIP expression in thymocytes
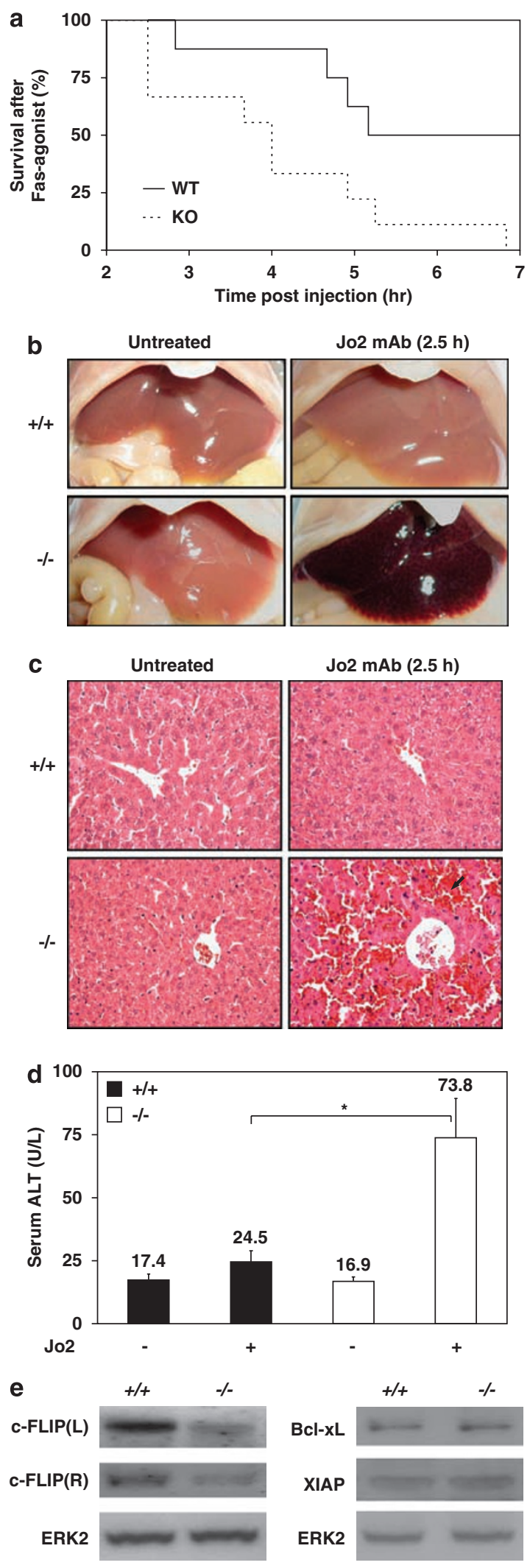

(Figure 5) and hepatocytes (Figure 6), we asked if c-FLIP expression level was affected during BCR engagement of CD40-activated faim $^{-1-} \mathrm{B}$ cells. As shown in Figure 7, both $c-F L I P(L)$ and $c-F L I P(R)$ expressions were increased in wild-type B cells activated with anti-CD40 mAb followed by concomitant treatment with anti-IgM antibody. By contrast, CD40-activated faim $^{-1-} \mathrm{B}$ cells did not show an increased expression of $c$-FLIP(L) and $c-F L I P(R)$ upon subsequent BCR engagement, which correlated well with their augmented apoptosis upon Fas treatment (Figure 3). At the same time, we also examined the expression levels of GADD45 $\beta$, XIAP and $\mathrm{Bcl}-\mathrm{xL}$, which are known to play antiapoptotic roles in activated $B$ cells, ${ }^{22-24}$ in wild-type and faim $^{-1-}$ CD40-activated B cells upon BCR stimulation. As shown in Figure 7, the expression level of GADD45 $\beta$ and $\mathrm{XIAP}$ were not induced whereas a $\mathrm{Bcl}-\mathrm{xL}$ expression was similarly induced by a BCR engagement in wild-type and faim $^{-1-}$ B cells. Taken together, these results suggest that FAIM plays a specific role in the induction of c-FLIP during BCR stimulation, which could act to protect CD40-activated B cells from Fas-mediated apoptosis.

\section{Discussion}

We have inactivated the murine faim gene and found faim $^{-1-}$ mice to be viable. The mutant mice also showed no gross defect in B- and T-cell development. Thus, the phenotype of faim $^{-1-}$ mice contrasted with other mouse mutants in apoptosis such as $\mathrm{fadd}^{-1-}, \mathrm{c}$-flip ${ }^{-/-}$and caspase $^{-1-}$ mice, which suffered from embryonic lethality ${ }^{25-28}$ and with fas $^{-/-}$ mice which developed abnormal lymphoid populations and autoimmunity. ${ }^{4}$

Although FAIM does not play a role in lymphoid cell development, we show here that it does regulate the apoptosis of lymphocytes triggered through Fas. In the absence of FAIM, both B cells and thymocytes are more susceptible to Fas-triggered cell death (Figures 3 and 4). In addition, it appears that FAIM acts specifically in the Fasinduced cell death pathway but not in apoptotic pathways triggered by steroid, radiation or $\mathrm{TNF}-\alpha$ treatment.

Biochemical analyses of Fas-triggered apoptosis of faim $^{-1}$ thymocytes indicate that the lack of FAIM leads to the enhanced activation of caspases- 8 and -3 (Figure 4). As caspase- 8 is part of the DISC, we have examined the expression of its components and found no difference in the protein expression level of Fas, FADD and caspase- 8 in faim $^{-1-}$ thymocytes

Figure 6 Enhanced sensitivity to Fas-induced lethality and liver failure in faim $^{-1-}$ mice. (a) Survival curves of wiltype $(n=8)$ and faim ${ }^{-1-}(n=9)$ mice injected i.p. with $10 \mu \mathrm{g}$ of an agonistic anti-Fas mAb (Jo2) indicated that death was increased in the mutant compared with wild-type mice, $P=0.015\left(\chi^{2}\right.$ test). Thorough analysis revealed no difference ( $P=0.90$, Student's $t$-test) in body weight between wild-type $(21.90 \pm 1.48 \mathrm{~g})$ and FAIM-deficient $(21.76 \pm 1.29 \mathrm{~g})$ mice. (b) Macroscopic appearance of livers at $2.5 \mathrm{~h}$ after anti-Fas mAb injection. Wild-type $(n=16)$ and faim $^{-1-}(n=13)$ mice were injected with Jo2 mAb and were killed for liver examination prior to the occurrence of death. (c) Representative hematoxylineosin staining of liver sections at $2.5 \mathrm{~h}$ after anti-Fas mAb injection. (d) Serum levels of ALT in wild-type $(n=12)$ and faim ${ }^{-1-}(n=15)$ mice at $2.5 \mathrm{~h}$ after PBS or anti-Fas mAb injection, ${ }^{*} P=0.0097$, (Two-side Student's $t$-test). (e) Reduced $c$-FLIP $(L)$ and $c$-FLIP(R) expression in faim ${ }^{-1-}$ liver sample. Anti-ERK2 blots were used as loading controls. Data are representative of 4-8 independent experiments 


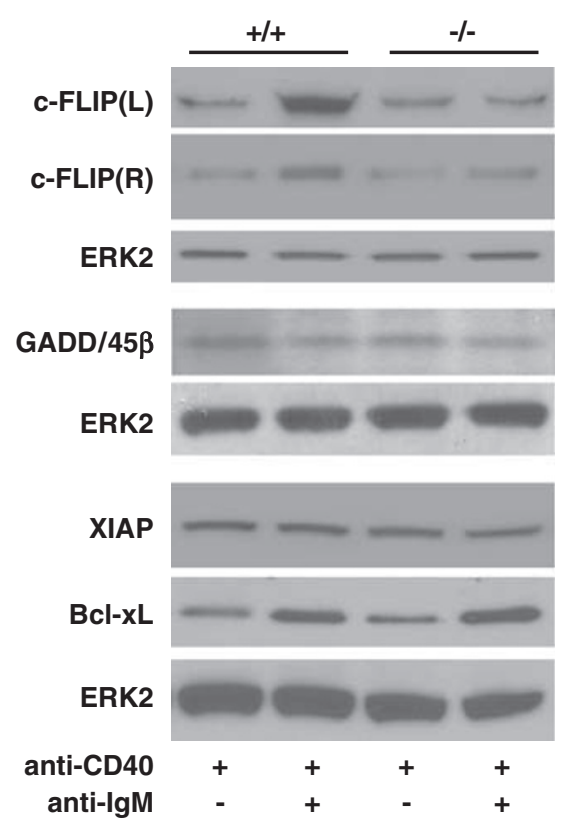

Figure 7 Expression levels of various anti-apoptotic molecules in anti-CD40treated and anti-CD40 plus anti-lgM-treated wild-type and faim ${ }^{-1-} \mathrm{B}$ cells. Western blot analysis showing c-FLIP(L), c-FLIP(R), GADD45 $\beta, \mathrm{Bcl}-\mathrm{xL}$, and XIAP protein expression in anti-CD40-activated wild-type $B$ cells treated with anti-IgM antibody over a non-treated control. The anti-ERK2 blot served as a loading control. Data shown are representative of 3-4 independent experiments

compared with wild-type control. Interestingly, the expression level of $c$-FLIP $(L)$ and c-FLIP $(R)$ were very much reduced in the mutant thymocytes. As c-FLIP plays an inhibitory role in Fasinduced apoptosis, the reduced level of c-FLIP expression in faim $^{-1-}$ thymocytes could partially explain the heightened sensitivity of these mutant thymocytes to Fas-triggered apoptosis. It has been well documented that c-FLIP antagonizes Fas-induced apoptosis by interfering with the recruitment of caspase- 8 to the death receptor. ${ }^{6}$ Indeed, we found that the amount of caspase-8 that could co-immunoprecipitate with Fas was significantly increased upon Fas-activation in faim $^{-1-}$ thymocytes compared with wild-type control (Figure 5). The increased association of caspase-8 with Fas in faim $^{-1-}$ thymocytes upon Fas-activation also helps to explain the enhanced sensitivity of the mutant cells to Fas-triggered cell death. Because the amount of caspase- 8 that was associated with Fas was found to be similar between wild-type and mutant thymocytes before Fas engagement, but was increased in mutant thymocytes upon Fas-activation, one could infer that under normal physiological conditions FAIM could act to modulate Fas-signaling by regulating C-FLIP expression and controlling the amount of caspase- 8 that could associate with Fas. This arrangement serves to set a threshold for the full activation of Fas and to prevent background or accidental signaling that could lead to the irreversible state of apoptosis.

Our in vivo experiment suggested that besides lymphocytes, FAIM could also play a role in modulating the apoptosis of other cell types such as hepatocytes. This was evident as the in vivo administration of the agonistic anti-Fas antibody led to massive liver damage and accelerated lethality in faim ${ }^{-1-}$ mice. Thus because of its protective role in the apoptosis of liver cells, FAIM could serve as a possible therapeutic target for the treatment of various forms of liver cirrhosis.

And consistent with our work on thymocytes, we also found c-FLIP $(L)$ and c-FLIP(R) expression levels to be perturbed in faim $^{-1-}$ hepatocytes. This further strengthens the hypothesis that a possible anti-apoptotic mechanism of FAIM is achieved through its regulation of the expression level of c-FLIP. Indeed, further experimentation also indicated that the protective effect of BCR signaling against Fas-mediated apoptosis of CD40activated $B$ cells is in part mediated through the upregulation of FAIM and leading to the increased expression of C-FLIP $(L)$ and $c-F L I P(R)$. Thus, the induction of FAIM and c-FLIP expressions appear to be a common mechanism shared by hepatocytes, thymocytes and B-lymphocytes in the protection against Fasmediated cell death.

Although we had shown that the expression level of FAIM and c-FLIP correlated with the sensitivity of hepatocytes and lymphocytes to Fas-mediated apoptosis, the finding that faim $^{-1-}$ thymocytes behaved similarly to wild-type thymocytes in their response to TNF- $\alpha$ induced apoptosis, which is also caspase-8 dependent, suggests that other molecules in addition to c-FLIP could also regulate the susceptibility of wild-type and faim $^{-1-}$ cells to TNF- $\alpha$ triggered apoptosis.

FAIM is expressed in neurons and has been shown to regulate neurite outgrowth ${ }^{8}$ and neuronal survival. ${ }^{9}$ It is possible that FAIM could play a more critical role in the nervous system and we are currently assessing its involvement in Parkinson's and Alzheimer's diseases where massive neuronal cell death is known to occur. The ability of FAIM to induce neurite outgrowth in addition to its ability to enhance neuronal survival also render it as an ideal therapeutic target for these diseases.

Finally, because FAIM could confer resistance to Fasinduced apoptosis of cells, its abnormal expression could render survival advantage to cells and may lead to tumor formation or autoimmunity. This possibility is also currently under investigation.

\section{Materials and Methods}

Generation of faim $^{-I-}$ mice. The CDNA for murine faim was used to probe mouse 129 genomic DNA library and this yielded a phage clone containing the first three exons of faim. Restriction enzyme digestion, Southern blotting and DNA sequencing were used to map the genomic locus. A targeting vector, designed to replace the first two exons with a neomycin-resistant gene, was linearized and transfected into E14.1 ES cells. G418-resistant cells were analyzed by Southern blotting with a $3^{\prime}$ external probe as indicated in Figure 1. Two homologous recombinants were injected into C57BL/6 blastocysts to generate chimeric mice for germline transmission of the inactivated allele. Mutant mice from two different ES cell clones exhibited comparable phenotypes. Mice in our experiments have mixed 129 and $\mathrm{C} 57 \mathrm{BL} / 6$ genetic background and were used according to national guidelines.

Flow cytometry analyses. Single-cell suspensions were obtained from thymus, spleen, lymph nodes and bone marrow of mice as described earlier, ${ }^{29}$ and stained with FITC-, PE- and biotin-conjugated antibodies to cell surface antigens. Biotin-conjugated antibody staining was revealed by second step StrepavidinCyChrome staining. The following antibodies from BD Pharmingen (San Diego, CA, USA) were used: anti-lgM (R6-60.2), anti-B220 (RA3-6B2), anti-CD3e (145-2C11), anti-CD4 (L3T4), anti-CD5 (53-7.3), anti-CD8 (53-6.7), anti-CD19 (1D3), anti-CD21 (7G6), anti-CD23 (B3B4), anti-CD25 (7D4), anti-CD43 (S7), anti-CD44 (IM7) and anti-CD62L (MEL-14). Data were collected on a FACScan (BD, Mountain View, CA, USA) and analyzed using CellQuest software. 
Fas-induced liver failure and histologic examination. Eight- to tenweek old $\mathrm{faim}^{-1-}$ and wild-type littermates with similar body weight were i.p. injected with $10 \mu \mathrm{g}$ of anti-Fas (Jo-2) antibody (BD Pharmingen) in $200 \mu \mathrm{l} \mathrm{PBS}$. Mice were either observed over a $10 \mathrm{~h}$ period for mortality or killed at matched time points for histology. Liver samples were fixed at $4{ }^{\circ} \mathrm{C}$ in $4 \%$ paraformaldehyde for $24 \mathrm{~h}$, dehydrated and paraffin-embedded. Liver sections of 5-micron thickness were stained with haematoxylin-eosin for histopathological evaluation of hepatic injury. Serum ALT was detected using the method of Reitman and Frankel (Span Diagnostics, India).

Apoptosis assay. Apoptosis was determined by propidium iodide (PI) staining as described by Nicoletti et al..$^{30}$ Briefly, thymocytes were treated with FasL $(10 \mathrm{ng} / \mathrm{ml}$ for $6 \mathrm{~h}), \mathrm{TNF}-\alpha(50 \mathrm{ng} / \mathrm{ml}$ with $100 \mu \mathrm{M}$ cycloheximide), DEX ( $1 \mu \mathrm{M}$ for $6 \mathrm{~h}$ ) or $\gamma$-irradiation for indicated times and fixed in ethanol. After fixation, cells were washed and resuspended in staining buffer $(20 \mu \mathrm{g} / \mathrm{ml} \mathrm{PI}, 200 \mu \mathrm{g} / \mathrm{ml}$ RNase and $0.1 \%$ Triton X-100 in PBS) for $1 \mathrm{~h}$ before FACS analysis. Results were analyzed with WinMDI software version 2.8 (Joseph Trotter, Scripps Research Institute). For B-cell apoptosis, cells were pre-activated with anti-CD40 mAb $(2 \mu \mathrm{g} / \mathrm{ml})$ for $48 \mathrm{~h}$, followed by treatment with FasL for $6 \mathrm{~h}$. In certain experiments, anti-CD40-stimulated $B$ cells were treated with anti-IgM antibodies $(10 \mu \mathrm{g} / \mathrm{ml})$ for $8 \mathrm{~h}$ prior to the addition of FasL.

Western blot analyses of protein expression. For Fas stimulation, thymocytes were incubated with $10 \mathrm{ng} / \mathrm{ml}$ of Fas ligand (Alexis) at $37^{\circ} \mathrm{C}$ for $6 \mathrm{~h}$. Whole-cell extracts were prepared using lysis buffer $(10 \mathrm{mM}$ Tris- $\mathrm{HCl}, \mathrm{pH}$ 8.0, $150 \mathrm{mM} \mathrm{NaCl}, 1 \mathrm{mM}$ EDTA, $1 \%$ Igepal CA-630, $0.2 \mathrm{mM} \mathrm{Na}_{3} \mathrm{VO}_{4}$ and a Protease Inhibitor Cocktail (Roche)). Protein concentration was measured by a colorimetric assay (Bio-Rad) and equal amount of proteins were loaded onto SDS gels. After transfer to PVDF membranes, the proteins were probed with primary antibodies $(1 \mu \mathrm{g} / \mathrm{ml})$, followed by horseradish peroxidase-conjugated secondary antibodies, washed and visualized with SuperSignal West Pico/Dura chemiluminescent substrate (Pierce). The blots were reprobed with an ERK2specific antibody to provide loading control. Antibodies used were as follows: from Santa Cruz: rabbit anti-ERK2 (C-14), mouse anti-caspase-8 p20 (D-8), rabbit anti-PARP (H250), rabbit anti-FADD (H181), rabbit anti-c-FLIP $/ \mathrm{L}$ (H202), rabbit anti-XIAP (H-202), rabbit anti-Bcl- $\mathrm{x}_{\mathrm{S} / \mathrm{L}}(\mathrm{M}-125)$, rabbit anti- $\kappa_{\kappa} \mathrm{B}-\alpha(\mathrm{C}-21)$, rabbit antiGADD45 $\beta(\mathrm{H}-70)$ and rabbit anti-FAS (M-20); from Cell Signaling: rabbit anticleaved caspase-3 (Asp175) antibody and rabbit anti-phospho-NF-kappa B p65 (ser536)(93H1). The FAIM rabbit polyclonal antibody was raised in-house against the full-length protein of mouse FAIM.

Immunoprecipitation. For immunoprecipitation studies, anti-Fas antibody or anti-caspase-8 antibody was coupled to Protein A/G Plus-Agarose (Santa Cruz) at $4{ }^{\circ} \mathrm{C}$ overnight. The beads were washed three times in lysis buffer and incubated with pre-cleared total cell lysates of untreated or FasL-treated samples overnight at $4{ }^{\circ} \mathrm{C}$. Subsequently, the beads were washed three times in lysis buffer and boiled in loading buffer for $5 \mathrm{~min}$. Released proteins were resolved in $10 \%$ SDSpolyacrylamide gel and blotted onto polyvinylidene difluoride membranes. The membranes were first probed with anti-caspase-8 or anti-Fas antibody and subsequently with the immunoprecipitating antibodies.

Statistical analysis. All experiments were performed at least three times. Data are presented as mean \pm S.E.M. Statistical comparison of the data was performed using Student's t-test. Group difference with $P<0.05$ was considered statistically significant. The survival curve obtained from the Kaplan-Meier procedure was analysed using $\chi^{2}$ test, and $P<0.05$ was considered statistically significant.

Acknowledgements. This work was supported by the Biomedical Research Council of the Agency for Science, Technology and Research, Singapore. We thank members of the laboratory for insightful discussion and staff of the Biological Resource Center for the care and maintenance of mice.

1. Nagata S. Apoptosis regulated by a death factor and its receptor: Fas ligand and Fas. Philos Trans R Soc Lond B Biol Sci 1994; 345: 281-287.

2. Krammer PH. CD95's deadly mission in the immune system. Nature 2000; 407: 789-795.

3. Takahashi T, Tanaka M, Brannan Cl, Jenkins NA, Copeland NG, Suda T et al. Generalized lymphoproliferative disease in mice, caused by a point mutation in the Fas ligand. Cell 1994; 76: 969-976.
4. Watanabe-Fukunaga $\mathrm{R}$, Brannan $\mathrm{Cl}$, Copeland NG, Jenkins NA, Nagata $S$. Lymphoproliferation disorder in mice explained by defects in Fas antigen that mediates apoptosis. Nature 1992; 356: 314-317.

5. Kischkel FC, Hellbardt S, Behrmann I, Germer M, Pawlita M, Krammer PH et al. Cytotoxicity-dependent APO-1 (Fas/CD95)-associated proteins form a death-inducing signaling complex (DISC) with the receptor. EMBO J 1995; 14: 5579-5588.

6. Scaffidi C, Schmitz I, Krammer PH, Peter ME. The role of c-FLIP in modulation of CD95-induced apoptosis. J Biol Chem 1999; 274: 1541-1548.

7. Schneider TJ, Fischer GM, Donohoe TJ, Colarusso TP, Rothstein TL. A novel gene coding for a Fas apoptosis inhibitory molecule (FAIM) isolated from inducibly Fas-resistant B lymphocytes. J Exp Med 1999; 189: 949-956.

8. Sole C, Dolcet X, Segura MF, Gutierrez H, Diaz-Meco MT, Gozzelino R et al. The death receptor antagonist FAIM promotes neurite outgrowth by a mechanism that depends on ERK and NF-kapp B signaling. J Cell Biol 2004; 167: 479-492.

9. Segura MF, Sole C, Pascual M, Moubarak RS, Perez-Garcia MJ, Gozzelino R et al. The long form of Fas apoptotic inhibitory molecule is expressed specifically in neurons and protects them against death receptor-triggered apoptosis. J Neurosci 2007; 27: 11228-11241.

10. Jaluria $P, C h u C$, Betenbaugh $M$, Shiloach J. Cells by design: a mini-review of targeting cell engineering using DNA microarrays. Mol Biotechnol 2008; 39: 105-111.

11. Wong DC, Wong KT, Nissom PM, Heng CK, Yap MG. Targeting early apoptotic genes in batch and fed-batch $\mathrm{CHO}$ cell cultures. Biotechnol Bioeng 2006; 95: 350-361.

12. Rothstein TL, Wang JK, Panka DJ, Foote LC, Wang Z, Stanger B et al. Protection against Fas-dependent Th1-mediated apoptosis by antigen receptor engagement in B cells. Nature 1995; 374: 163-165.

13. Lagresle C, Mondiere P, Bella C, Krammer PH, Defrance T. Concurrent engagement of $\mathrm{CD} 40$ and the antigen receptor protects naive and memory human B cells from APO-1/Fasmediated apoptosis. J Exp Med 1996; 183: 1377-1388.

14. Ogasawara J, Suda T, Nagata S. Selective apoptosis of CD4+CD8+ thymocytes by the anti-Fas antibody. J Exp Med 1995; 181: 485-491.

15. Hernandez-Caselles T, Stutman O. Immune functions of tumor necrosis factor. I. Tumor necrosis factor induces apoptosis of mouse thymocytes and can also stimulate or inhibit IL-6-induced proliferation depending on the concentration of mitogenic costimulation. J Immunol 1993; 151: 3999-4012.

16. Matylevich NP, Korol' BA, Nelipovich PA, Afanas'ev VN, Umanskii SR. D2O inhibition of interphase thymocyte death. Radiobiologiia 1991; 31: 27-32.

17. Fernandes-Alnemri T, Armstrong RC, Krebs J, Srinivasula SM, Wang L, Bullrich F et al. In vitro activation of CPP32 and Mch3 by Mch4, a novel human apoptotic cysteine protease containing two FADD-like domains. Proc Natl Acad Sci USA 1996; 93: 7464-7469.

18. Ueffing N, Keil E, Freund C, Kuhne R, Schulze-Osthoff K, Schmitz I. Mutational analyses of c-FLIPR, the only murine short FLIP isoform, reveal requirements for DISC recruitment. Cell Death Differ 2008; 15: 773-782.

19. Wang X, Zhang J, Kim HP, Wang Y, Choi AM, Ryter SW. Bcl-XL disrupts death-inducing signal complex formation in plasma membrane induced by hypoxia/reoxygenation. FASEB J 2004; 18: 1826-1833.

20. Lavrik IN, Mock T, Golks A, Hoffmann JC, Baumann S, Krammer PH. CD95 stimulation results in the formation of a novel death effector domain protein-containing complex. J Biol Chem 2008; 283: 26401-26408.

21. Ogasawara J, Watanabe-Fukunaga R, Adachi M, Matsuzawa A, Kasugai T, Kitamura $Y$ et al. Lethal effect of the anti-Fas antibody in mice. Nature 1993; 364: 806-809.

22. Kitada S, Zapata JM, Andreeff M, Reed JC. Bryostatin and CD40-ligand enhance apoptosis resistance and induce expression of cell survival genes in B-cell chronic lymphocytic leukaemia. Br J Haematol 1999; 106: 995-1004.

23. Rothstein TL, Zhong X, Schram BR, Negm RS, Donohoe TJ, Cabral DS et al. Receptorspecific regulation of B-cell susceptibility to Fas-mediated apoptosis and a novel Fas apoptosis inhibitory molecule. Immunol Rev 2000; 176: 116-133.

24. Zazzeroni F, Papa S, Algeciras-Schimnich A, Alvarez K, Melis T, Bubici C et al. Gadd45 beta mediates the protective effects of $\mathrm{CD} 40$ costimulation against Fas-induced apoptosis. Blood 2003; 102: 3270-3279.

25. Zhang J, Cado D, Chen A, Kabra NH, Winoto A. Fas-mediated apoptosis and activationinduced T-cell proliferation are defective in mice lacking FADD/Mort1. Nature 1998; 392 296-300.

26. Yeh WC, Pompa JL, McCurrach ME, Shu HB, Elia AJ, Shahinian A et al. FADD: essential for embryo development and signaling from some, but not all, inducers of apoptosis. Science 1998; 279: 1954-1958.

27. Yeh WC, Itie A, Elia AJ, Ng M, Shu HB, Wakeham A et al. Requirement for Casper (c-FLIP) in regulation of death receptor-induced apoptosis and embryonic development. Immunity 2000; 12: 633-642.

28. Varfolomeev EE, Schuchmann M, Luria V, Chiannilkulchai N, Beckmann JS, Mett IL et al. Targeted disruption of the mouse Caspase 8 gene ablates cell death induction by the TNF receptors, Fas/Apo1, and DR3 and is lethal prenatally. Immunity 1998; 9: 267-276.

29. Xu S, Huo J, Tan JE, Lam KP. Cbp deficiency alters Csk localization in lipid rafts but does not affect T-cell development. Mol Cell Biol 2005; 25: 8486-8495.

30. Nicoletti I, Migliorati G, Pagliacci MC, Grignani F, Riccardi C. A rapid and simple method for measuring thymocyte apoptosis by propidium iodide staining and flow cytometry. J Immunol Methods 1991; 139: 271-279. 